\title{
Diversidad genética de gallinas criollas en valles centrales de Oaxaca usando marcadores microsatélites
}

Héctor Luis-Chincoya ${ }^{\text {a }}$

José Guadalupe Herrera-Haro ${ }^{\text {a }}$

Amalio Santacruz-Varela ${ }^{b^{*}}$

Martha Patricia Jerez-Salas ${ }^{c}$

Alfonso Hernández-Garay ${ }^{a}$

a Colegio de Postgraduados.' Campus Montecillos. Recursos Genéticos y ProductividadGanadería. Texcoco, México.

${ }^{\mathrm{b}}$ Colegio de Postgraduados. Campus Montecillos. Recursos Genéticos y ProductividadGenética. Texcoco, México.

c Instituto Tecnológico del Valle de Oaxaca. Xoxocotlán, Oaxaca, México.

*Autor de correspondencia: asvarela@colpos.mx

\section{Resumen:}

Las poblaciones de gallinas criollas en explotaciones en pequeña escala poseen una gran diversidad y son parte del reservorio genético avícola en México; además, constituyen una importante fuente de proteína para las familias del medio rural. Con el propósito de conocer la variabilidad genética de poblaciones de gallinas criollas en la región central de Oaxaca, se tomaron muestras de sangre de 109 gallinas criollas, provenientes de 17 poblaciones y de 30 gallinas Plymouth Rock como testigos. Se usaron 10 marcadores de tipo microsatélites, con los que se detectó un total de 109 alelos, con $10.9 \pm 3.1$ alelos por locus en promedio. La heterocigosidad observada (Ho) varió de 0.575 (San Lucas) a 0.750 (San Antonino 2) y la esperada (He) de 0.625 (Testigo) a 0.733 (Huixtepec 2). A nivel general se encontró un incremento en el número de heterocigotos evidenciado por un nivel de endogamia global 
( $\left.\mathrm{F}_{\mathrm{IT}}\right)$ de 0.042 ; el índice $\mathrm{F}_{\mathrm{ST}}$ de diferenciación entre poblaciones fue moderado (0.059) y la endogamia de individuos dentro de poblaciones (FIS) resultó negativa (-0.017), lo que indica un exceso de heterocigotos a ese nivel. El análisis de conglomerados agrupó a las poblaciones de Nazareno, ITVO, San Lucas y San Antonio, evidenciando que son poblaciones de gallinas criollas aisladas y diferenciadas genéticamente en algunas características. Esta información es importante para diseñar futuros programas de conservación, selección y multiplicación de esta especie a nivel de traspatio en la región central de Oaxaca, México.

Palabras clave: Aves locales, SSR, Diversidad genética, Recursos zoogenéticos.

Recibido:16/10/2018

Aceptado: 03/02/2020

\section{Introducción}

El desarrollo de sistemas de producción avícola intensivos, altamente eficientes y rentables, aunado a la exigencia del mercado de productos de buena calidad y uniformes en la carne y huevo, propicia la incorporación de razas especializadas con alto rendimiento en estas características, provocando la disminución y erosión genética de los genotipos locales, denominados "criollos" por la disminución de su tamaño efectivo de población ${ }^{(1)}$. Estos genotipos criollos son explotados en forma rústica en unidades de producción de menor escala, adaptados al clima y a sistemas de producción de bajos insumos, en condiciones de bienestar y tolerantes a enfermedades ${ }^{(2)}$, además de formar parte del patrimonio cultural de las comunidades rurales. Por ello, la $\mathrm{FAO}^{(3)}$ señala la importancia que tiene para la humanidad la identificación y conservación de las gallinas locales dándoles atención especial a su mejora y conservación ${ }^{(4)}$, las cuales por su diversidad biológica presentan cambios continuos debido a los procesos de selección natural y migración.

Los microsatélites son marcadores moleculares para evaluar la variabilidad genética a nivel de ADN, basados en repeticiones en tándem de una a seis bases y estos son ampliamente utilizados por su distribución al azar en el genoma, alto polimorfismo y herencia codominante ${ }^{(5,6)}$. Mediante esta técnica se obtienen estadísticas genéticas descriptivas como heterocigosidad, distancia genética, número de alelos efectivos y contenido de información polimórfica de los marcadores entre poblaciones estrechamente relacionadas.

En México, los estudios realizados en la avicultura en pequeña escala con gallinas criollas, que aportan información sobre su diversidad genética basada en marcadores moleculares son escasos. En consecuencia, surge la necesidad de identificar las poblaciones avícolas 
existentes y desarrollar programas para su mejora y conservación que beneficien a los productores que viven en zonas rurales ${ }^{(7)}$. El objetivo de la investigación fue determinar la diversidad genética de poblaciones de gallinas criollas en la región de Valles Centrales, Oaxaca.

\section{Material y métodos}

\section{Toma de muestras}

La colección de muestras se realizó durante enero-diciembre de 2015, se utilizó un muestreo probabilístico por conglomerados en dos etapas ${ }^{(8)}$. Las unidades primarias las constituyeron los siete distritos y como unidades secundarias las unidades de producción (UP) dentro de los distritos. Se escogió una muestra de tres unidades primarias (n) y dentro de las unidades primarias se eligieron aleatoriamente un total de 17 unidades secundarias. En cada unidad secundaria (UP) se eligieron 6 gallinas, constituyendo un tamaño de muestra total de 109 gallinas adultas de las cuales: 93 gallinas criollas adultas, en primera etapa de postura, con peso promedio de $2.0 \mathrm{~kg}, 16$ gallos adultos. El inventario estimado de gallinas adultas fue de 2,004 animales para la región de Valles Centrales, lo que constituyó el 6 \% de la población muestreada. El tamaño de muestra fue obtenido con una precisión del $10 \%$ y confiabilidad del $90 \%$. El muestreo fue distribuido en seis núcleos geográficos (ITVO: Nazareno e Itvo; Cuilápam: San Antonio, San Lucas y Cuilápam; Etla: San Juan y Suchilquitongo; Huixtepec: Huixtepec 1, 2, 3 y 4; Ocotlán: Chichicapam, San Antonino 1 y 2; y Tlacolula: Teotitlán, Totolapam 1 y 2, más un Testigo de gallinas Plymouth Rock. A cada animal de la vena cubital del ala se le extrajeron $2 \mathrm{ml}$ de sangre, enseguida la sangre se depositó en tubos vacutainer con anticoagulante EDTA, conservándose a una temperatura de $-20{ }^{\circ} \mathrm{C}$ hasta su procesamiento en el laboratorio.

\section{Extracción de ADN, marcadores microsatélites y procedimiento de PCR}

El ADN se extrajo con el kit comercial ChargeSwitch® gDNA Plant Kit (Invitrogen), usando el protocolo del proveedor. La cuantificación del ADN se realizó con un espectrofotómetro de ultra-bajo volumen (NanoDrop 2000, Thermo Scientific, Wilmington, DE, USA) ajustando las concentraciones de ADN a $10 \mathrm{ng} \mu^{-1}$. Se utilizaron 10 pares de iniciadores de microsatélites para evaluar diversidad genética (Cuadro 1) reportados previamente ${ }^{(9-11)}$. Los iniciadores se marcaron con las etiquetas fluorescentes (6-FAM, HEX o ROX) en el extremo 5' para su detección por PCR múltiplex. 
Cuadro 1: Descripción de los iniciadores de los loci de microsatélites utilizados, temperatura de alineamiento $(\mathrm{Tm})$, tamaño de fragmento esperado

\begin{tabular}{|c|c|c|c|c|}
\hline Iniciador & Secuencia $5^{\prime}$ - 3' & $\begin{array}{l}\mathbf{T m} \\
\left({ }^{\circ} \mathbf{C}\right)\end{array}$ & $\begin{array}{c}\text { Tamaño } \\
\text { (pb) }\end{array}$ & Referencia \\
\hline MCW32-F & AGTTCCTTGTACAATTGTTA & 53.6 & 273 a 314 & h \\
\hline MCW32-R & TCATTACTAGTACAATCAAGATGG & & & 0 \\
\hline MCW68-F & CCTCACTGTGTAGTGTGGTAGTCA & 62 & 171 a 193 & \\
\hline MCW68-R & GAGAAGCTTGAACCTACCAGTCTT & & & $\mathrm{c}$ \\
\hline MCW94-F & GGAGCTGGTATTTGTCCTAAG & 53.6 & 77 a 95 & \\
\hline MCW94-R & GCACAGCCTTTTGACATGTAC & & & a \\
\hline MCW95-F & GATCAAAACATGAGAGACGAAG & 62 & 72 a 91 & \\
\hline MCW95-R & TTCATAGCTTGAATTGCATAGC & & & a \\
\hline MCW114- & AGCAA ACTGCTCAGTGCTGTG & 62 & 261 a 293 & \\
\hline MCW114- & & & & $\mathrm{c}$ \\
\hline $\mathrm{R}$ & GCGTTGAAAGTAGTGCTTCCG & & & \\
\hline $\begin{array}{l}\text { MCW131- } \\
\text { F } \\
\text { MCW131- }\end{array}$ & GTTGCTGATTCTAAGGCAGGC & 53.6 & 195 a 217 & $\mathrm{c}$ \\
\hline $\mathrm{R}$ & TTGCAGTTGTAAAGGTGTAGC & & & \\
\hline MCW134- & & 62 & 260 a 284 & \\
\hline $\begin{array}{l}\text { F } \\
\text { MCW134- }\end{array}$ & GGAGACTTCATTGTGTAGCAC & & & $\mathrm{b}$ \\
\hline $\mathrm{R}$ & ACCAAAAGACTGGAGGTCAAC & & & \\
\hline $\begin{array}{l}\text { MCW135- } \\
\text { F }\end{array}$ & ATATGCTGCAGAGGGCAGTAG & 62 & 124 a 150 & \\
\hline MCW135- & & & & $\mathrm{a}$ \\
\hline $\mathrm{R}$ & CATGTTCTGCATTATTGCTCC & & & \\
\hline MCW145- & & 62 & 164 a 212 & \\
\hline $\begin{array}{l}\text { F } \\
\text { MCW145- }\end{array}$ & ACTTTATTCTCCAAATTTGGCT & & & $\mathrm{b}$ \\
\hline $\mathrm{R}$ & AAACACAATGGCAACGGAAAC & & & \\
\hline MCW158- & & 53.6 & 164 a 224 & \\
\hline $\begin{array}{l}\text { F } \\
\text { MCW158- }\end{array}$ & GATCCATTTATAAAGACCCCAC & & & $\mathrm{a}$ \\
\hline $\mathrm{R}$ & TTCAATACTCCTTTGTAAAGCA & & & \\
\hline
\end{tabular}


El procedimiento de PCR constó de un volumen total de $25 \mu$ l que contenían: $5 \mu$ l de Buffer 5X (Promega), $2 \mu \mathrm{l}$ a $25 \mathrm{mM}$ de $\mathrm{MgCl}_{2}$ (Promega), $0.5 \mu \mathrm{l}$ mezcla de DNTPs (Promega) a 2 $\mathrm{mM}, 1 \mu \mathrm{l}$ de cada iniciador ( $5 \mathrm{pmol}$ ), $1 \mathrm{U}$ de Taq polimerasa (Promega) y $1 \mu \mathrm{l}$ de ADN molde (10 ng $\left.\mu \mathrm{l}^{-1}\right)$. La amplificación se realizó en un termociclador (Bio-Rad $\mathrm{C} 1000^{\mathrm{TM}}$ ) con las siguientes condiciones: desnaturalización inicial a $94{ }^{\circ} \mathrm{C}$ por $5 \mathrm{~min}$, seguido de 35 ciclos de desnaturalización de $94{ }^{\circ} \mathrm{C}$ por $45 \mathrm{seg}$, alineación para grupos de iniciadores a $53.6{ }^{\circ} \mathrm{C}$, $56.6{ }^{\circ} \mathrm{C}$ y $62{ }^{\circ} \mathrm{C}$ durante $1 \mathrm{~min}$, una extensión de $72{ }^{\circ} \mathrm{C}$ por $1 \mathrm{~min}$, y una extensión final a $72{ }^{\circ} \mathrm{C}$ durante $10 \mathrm{~min}$. Los productos de PCR fueron separados por electroforesis capilar en un secuenciador automático de ADN (Genetic Analyzer ABI-3130 Applied Biosytems, Foster City, CA, USA) y analizados con el programa GeneMapper® de Applied Biosystems.

\section{Análisis estadístico}

Con base en el perfil alélico determinado en cada individuo para cada locus, se calcularon las frecuencias alélicas, heterocigosidad observada (Ho) y heterocigosidad esperada (He), así como el equilibrio Hardy-Weinberg. Con base en el grado de diferenciación genética de las poblaciones, se estimó con los estadísticos F de Wright (FIS; FIT y F FT), considerando como criterio de agrupación los núcleos geográficos de origen de los individuos, para definir los parámetros de diversidad genética, se usó el programa POPGENE (Versión 1.3.2). Adicionalmente con las frecuencias alélicas de cada loci de las poblaciones, se realizó un análisis de componentes principales con el paquete SAS V. 9.4 ${ }^{(12)}$ y un análisis de agrupamiento por el método de UPGMA basado en distancias euclidianas para las poblaciones de gallinas criollas y grupo de referencia, para determinar su patrón de similitud genética.

\section{Resultados y discusión}

\section{Diversidad genética}

Se identificaron en total 109 alelos en las 18 poblaciones de gallinas, considerando los 10 loci evaluados, el número de alelos varió desde seis para MCW145 hasta 16 para MCW158, con un promedio de 10.9 alelos por locus; todos los loci fueron polimórficos (Cuadro 2) coincidiendo en algunos de los iniciadores en un mapeo en pollos ${ }^{(9)}$. El MCW145, fue la excepción porque presentó un menor valor con respecto a lo reportado previamente ${ }^{(9,10)}$. 
Cuadro 2: Parámetros de diversidad genética detectados con los de 10 loci microsatélites evaluados en 109 gallinas criollas distribuidas en 17 poblaciones

\begin{tabular}{|c|c|c|c|c|c|}
\hline Loci & $\mathbf{R a}$ & $\mathbf{N a}$ & $\mathrm{Ne}$ & Ho & $\mathbf{H e}$ \\
\hline MCW131 & 7 & 8 & 2.493 & 0.596 & 0.601 \\
\hline MCW158 & 8 & 16 & 8.682 & 0.525 & 0.888 \\
\hline MCW32 & 6 & 13 & 5.175 & 0.877 & 0.809 \\
\hline MCW94 & 7 & 10 & 5.170 & 0.912 & 0.809 \\
\hline MCW114 & 7 & 11 & 3.748 & 0.590 & 0.736 \\
\hline MCW134 & 9 & 12 & 5.065 & 0.927 & 0.805 \\
\hline MCW135 & 7 & 15 & 10.982 & 0.845 & 0.912 \\
\hline MCW145 & 8 & 6 & 2.210 & 0.080 & 0.549 \\
\hline MCW68 & 7 & 9 & 4.035 & 0.749 & 0.755 \\
\hline MCW95 & 7 & 9 & 2.176 & 0.476 & 0.542 \\
\hline Media & & 10.9 & 4.974 & 0.658 & 0.738 \\
\hline Desviación estándar & & 3.142 & 2.866 & 0.133 & 0.133 \\
\hline
\end{tabular}

En investigaciones realizadas en diferentes razas locales de gallinas criollas, encontraron un número total de alelos por locus menor al presente estudio, utilizando diferentes loci de microsatélites aplicados en la misma especie. Así, en Suecia, Alemania y Polonia, se reportaron 113, 217 y 62 alelos observados con 24, 29 y 10 loci respectivamente ${ }^{(1,13,14)}$; por otro lado en China, Tailandia e India se encontraron valores similares 276, 227 y 170 alelos con 29, 20 y 17 loci $^{(6,15,16)}$. De igual manera, investigadores de Israel e Irán ${ }^{(17,18)}$ encontraron 211 y 310 alelos en poblaciones locales, usando 22 y 31 loci de microsatélites, respectivamente. Coincidiendo en un promedio similar al encontrado en este estudio. El numero promedio de alelos reportados en las poblaciones de Oaxaca, México, muestran una amplia diversidad de genes, la cual es similar a la de otros países que poseen razas de gallinas locales bien definidas.

El número de alelos efectivos por locus varió desde 2.1 (MCW95) hasta 10.9 (MCW135), con un promedio de 4.9. El valor de heterocigosidad observada (Ho) osciló de 0.08 con el locus MCW145, y el valor más alto fue de 0.92 en el locus MCW134 (Cuadro 2), mientras que la heterocigosidad esperada (He) presentó valores de 0.54 (MCW95 y MCW145) hasta 0.91 (MCW135). Si bien esto sugiere la existencia de una amplia diversidad genética de las poblaciones analizadas, también indica discrepancias entre ambos tipos de heterocigosidad, lo que indica desviaciones del equilibrio de Hardy-Weinberg, siendo las causales los procesos de migración, que ocurren en las poblaciones estudiadas o la deriva genética, posiblemente atribuida al bajo número de individuos que normalmente componen cada una de las 
poblaciones. Otros estudios ${ }^{(10,19)}$ se han reportado valores de He para el locus MCW145 de 0.69 y 0.75 , respectivamente; estos resultados fueron mayores a lo encontrado en este estudio (0.54), debido a que este locus se asocia con un menor número de alelos, que influyen de manera importante en el valor, pues cada alelo representa un término adicional a la sumatoria para el cálculo de He. En estudios ${ }^{(10,20)}$ para el loci MCW134 se reportaron valores de $\mathrm{He}=0.68$ y 0.64 en China, respectivamente. Son valores más bajos en referencia al valor obtenido en este estudio (0.805).

El número de alelos encontrados en poblaciones por loci fueron en orden descendente: el genotipo de referencia Plymouth Rock, con 70 alelos, seguida de Cuilápam con 65 alelos, contrastando con la población Teotitlán con 41 alelos, siendo el valor más bajo (Cuadro 3). Sin embargo, la población San Antonino 1 presentó el mayor número de alelos efectivos (43.8), seguida de ITVO con 37.8 alelos, mientras la población de Suchilquitongo registró 29 alelos efectivos, lo que implica un alto número de alelos que puede ser transmitido a la siguiente generación de gallinas criollas.

Cuadro 3: Número de alelos totales (NTa) y alelos efectivos totales (NTe), heterocigosidad observada (Ho) y esperada (He) en las poblaciones estudiadas

\begin{tabular}{lccccc}
\hline \multicolumn{1}{c}{ Población } & Ni* $^{*}$ & NTa & NTe & Ho $\pm \mathbf{D E}$ & He $\pm \mathbf{D E}$ \\
\hline Nazareno & 7 & 49 & 35.288 & $0.577 \pm 0.296$ & $0.684 \pm 0.258$ \\
ITVO & 7 & 60 & 37.805 & $0.674 \pm 0.248$ & $0.692 \pm 0.229$ \\
San Antonio & 7 & 48 & 31.166 & $0.630 \pm 0.284$ & $0.643 \pm 0.234$ \\
San Lucas & 7 & 48 & 32.506 & $0.575 \pm 0.205$ & $0.732 \pm 0.098$ \\
Cuilápam & 7 & 65 & 39.618 & $0.615 \pm 0.311$ & $0.691 \pm 0.236$ \\
Plymouth & 30 & 70 & 37.639 & $0.631 \pm 0.297$ & $0.625 \pm 0.253$ \\
Rock & & & 34.564 & $0.633 \pm 0.227$ & $0.656 \pm 0.238$ \\
Chichicapam & 6 & 53 & 30.550 & $0.65 \pm 0.298$ & $0.654 \pm 0.252$ \\
Teotitlán & 6 & 41 & 32.539 & $0.587 \pm 0.304$ & $0.643 \pm 0.265$ \\
San Juan & 7 & 53 & 29.88 & $0.676 \pm 0.361$ & $0.661 \pm 0.112$ \\
Suchilquitongo & 7 & 53 & 43.851 & $0.690 \pm 0.332$ & $0.716 \pm 0.230$ \\
San Antonino 1 & 7 & 63 & 35.533 & $0.75 \pm 0.316$ & $0.695 \pm 0.266$ \\
San Antonino 2 & 6 & 45 & 34.179 & $0.67 \pm 0.316$ & $0.71 \pm 0.124$ \\
Huixtepec 1 & 7 & 58 & 36.984 & $0.711 \pm 0.262$ & $0.733 \pm 0.121$ \\
Huixtepec 2 & 7 & 53 & 34.482 & $0.7 \pm 0.247$ & $0.718 \pm 0.144$ \\
Huixtepec 3 & 7 & 52 & 36.009 & $0.716 \pm 0.324$ & $0.710 \pm 0.204$ \\
Huixtepec 4 & 6 & 46 & 32.729 & $0.673 \pm 0.337$ & $0.673 \pm 0.264$ \\
Totolapam 1 & 7 & 42 & 36.746 & $0.703 \pm 0.325$ & $0.681 \pm 0.222$ \\
Totolapam 2 & 8 & 57 & \multirow{2}{*}{ Población de refenc } & \\
\hline
\end{tabular}

†Población de referencia $\mathrm{Ni}^{*}=$ Número de individuos por población. 
La heterocigosidad observada (Ho) en las poblaciones de gallinas varió desde 0.575 en la población San Lucas hasta 0.75 en la población San Antonino 2, con promedio de 0.741, mientras que la heterocigosidad esperada (He) fue más baja en el grupo de referencia (0.625), lo cual sugiere que existe un nivel de parentesco menor entre progenitores. Así mismo, las poblaciones Huixtepec 2, San Lucas y San Antonino 1 (0.733, 0.732 y 0.716, respectivamente), poseen la mayor diversidad genética, y por consiguiente un mayor potencial para su utilización en programas de mejoramiento. En estudios anteriores en gallinas locales $^{(2,21-23)}$ realizados en Suecia, Corea, China e India se reportaron valores de Ho y He más bajos. Por otro lado, en poblaciones de gallinas locales del Tíbet y China, encontraron valores de 0.798 y 0.76 para $\mathrm{He}^{(10,24)}$, más elevados con respecto a lo encontrado en gallinas de Oaxaca, México; esto podria atribuirse al uso de diferentes loci de microsatelites.

\section{Diferenciación genética de poblaciones de gallinas}

Las agrupaciones de Ocotlán y Tlacolula presentaron los valores de FIS más bajos (-0.072 y -0.079), indicando un mayor número de individuos heterocigotos dentro de cada población. En general, las agrupaciones de gallinas criollas son poblaciones no consanguíneas, esto por los valores menores a cero; y cercanas al equilibrio de Hardy-Weinberg. Estudios han enfatizado la adaptación de las gallinas locales a las diferentes condiciones geográficas que existen en cada país y por ello el equilibrio Hardy-Weinberg se mantiene constante en cada generación $^{(2,21)}$.

En el caso de $\mathrm{F}_{\text {IT, }}$ los valores más bajos fueron para Ocotlán y el testigo (-0.003 y -0.037), a nivel de unidades de producción. Mientras en Ocotlán, como era de esperarse, debido al cambio constante de reproductores machos mediante compra directa en los mercados regionales, se genera un importante flujo de material genético avícola local a nivel regional. Mientras en el grupo testigo, por su origen y esquema de reproducción establecido, se maximiza la obtención de individuos heterocigotos. En este caso, el grupo de Cuilápam presentó los mayores valores para FIS $(0.106)$ y FIT $(0.177)$, con respecto al resto de las agrupaciones, pero estadísticamente, estableciendo intervalos de confianza, se encuentra en equilibrio de sus individuos heterocigotos (Cuadro 4), explicado por la falta de un proceso de sustitución de machos en las unidades de producción, aumentando la relación de parentesco y la proporción de loci homocigotos. 
Cuadro 4: Estadísticos F de Wright de 10 loci de microsatélites para siete grupos de gallinas y sus límites de confianza en Valles Centrales de Oaxaca

$\mathrm{F}_{\mathrm{IS}}$, indicador de endogamia para individuos dentro de poblaciones individuales; $\mathrm{F}_{\mathrm{IT}}$, indicador de

\begin{tabular}{lrllll}
\hline \multicolumn{1}{c}{ Poblaciones } & \multicolumn{1}{c}{ FIS } & \multicolumn{1}{c}{ LS y LI } & \multicolumn{1}{c}{ FIT $_{\text {IT }}$} & \multicolumn{1}{c}{ LS y LI } & F \\
\hline ITVO & 0.015 & $0.1424 \mathrm{a}-0.111$ & 0.091 & $0.209 \mathrm{a}-0.027$ & 0.077 \\
Cuilápam & 0.106 & $0.253 \mathrm{a}-0.040$ & 0.177 & $0.337 \mathrm{a} 0.017$ & 0.079 \\
Testigo & -0.037 & $0.090 \mathrm{a}-0.165$ & -0.037 & $0.090 \mathrm{a}-0.165$ & 0.000 \\
Etla & -0.014 & $0.248 \mathrm{a}-0.277$ & 0.032 & $0.288 \mathrm{a}-0.223$ & 0.046 \\
Huixtepec & -0.040 & $0.170 \mathrm{a}-0.250$ & 0.038 & $0.234 \mathrm{a}-0.156$ & 0.076 \\
Ocotlán & -0.072 & $0.076 \mathrm{a}-0.220$ & -0.003 & $0.138 \mathrm{a}-0.145$ & 0.063 \\
Tlacolula & -0.079 & $0.172 \mathrm{a}-0.330$ & -0.001 & $0.227 \mathrm{a}-0.229$ & 0.072 \\
\hline Global & -0.017 & & 0.042 & & 0.059 \\
\hline
\end{tabular}

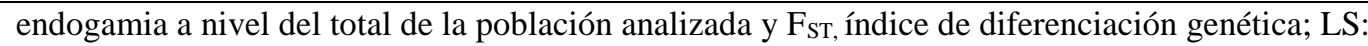

Límite superior y LI: Límite inferior.

Las agrupaciones de Cuilápam, ITVO y Huixtepec presentaron una diferenciación genética (FST $_{\text {SToderada }}{ }^{(25)}(0.079,0.077$ y 0.076 , respectivamente); estos resultados indican que existe una diferenciación entre las poblaciones por aislamiento geográfico o de manejo, y por consiguiente, un reducido flujo genético entre individuos de las distintas regiones avícolas. El valor global para $\mathrm{F}_{\mathrm{ST}}$ de 0.059 indica el $94.1 \%$ de la variación total está dentro las poblaciones y solamente el $5.9 \%$ se encuentra entre de las poblaciones ${ }^{(25)}$ (Cuadro 4). Confirmando una moderada diversidad genética dentro de las poblaciones, lo que se asocia con la forma de selección de machos reproductores y hembras de remplazo realizado por las productoras y demuestra el potencial que existe para realizar mejoramiento genético intrapoblacional a través de esquemas de selección recurrente que permite aprovechar los efectos de la selección y recombinación en un proceso continuo. Resultados similares en cuanto a la diferenciación genética han sido reportados ${ }^{(26-28)}$ en poblaciones de gallinas locales de Egipto, Bután, Asia y China, mientras que en otro estudio con ocho razas de pollos nativos de Corea ${ }^{(29)}$ reportaron un mayor coeficiente de diferenciación (0.180).

\section{Relaciones entre las poblaciones}

El análisis de componentes principales (CP) explicó un 32.2 \% de la variación total con los tres primeros componentes, conformados por alelos más importantes de los 10 loci de microsatélites (Cuadro 5). En el plano de tridimensionalidad de las poblaciones (Figura 1), con base en los primeros tres componentes principales, se identifica a las poblaciones más alejadas: Nazareno $=\mathrm{A}, \mathrm{ITVO}=\mathrm{B}$ y Suchiquitongo $=\mathrm{J}$ y Huixtepec $=\mathrm{N}$, caracterizadas por no permitir material genético avícola comercial, lo que sugiere un marcado flujo genético dentro de las mismas poblaciones. En otros estudios se han encontrado diferenciaciones claras en 
las poblaciones de gallinas locales en $\mathrm{Kenia}^{(30)}$, así como en la india ${ }^{(31)}$, usando CP considerando a los dos primeros componentes con valores de $46.25 \%$ y $23.37 \%$ de la variación genética con gallinas locales.

Figura 1: Plano de la dimensionalidad de las poblaciones de gallinas con los componentes principales CP1 vs $\mathrm{CP} 2$ vs $\mathrm{CP} 3$, generados con las frecuencias alélicas de 10 loci de microsatélites

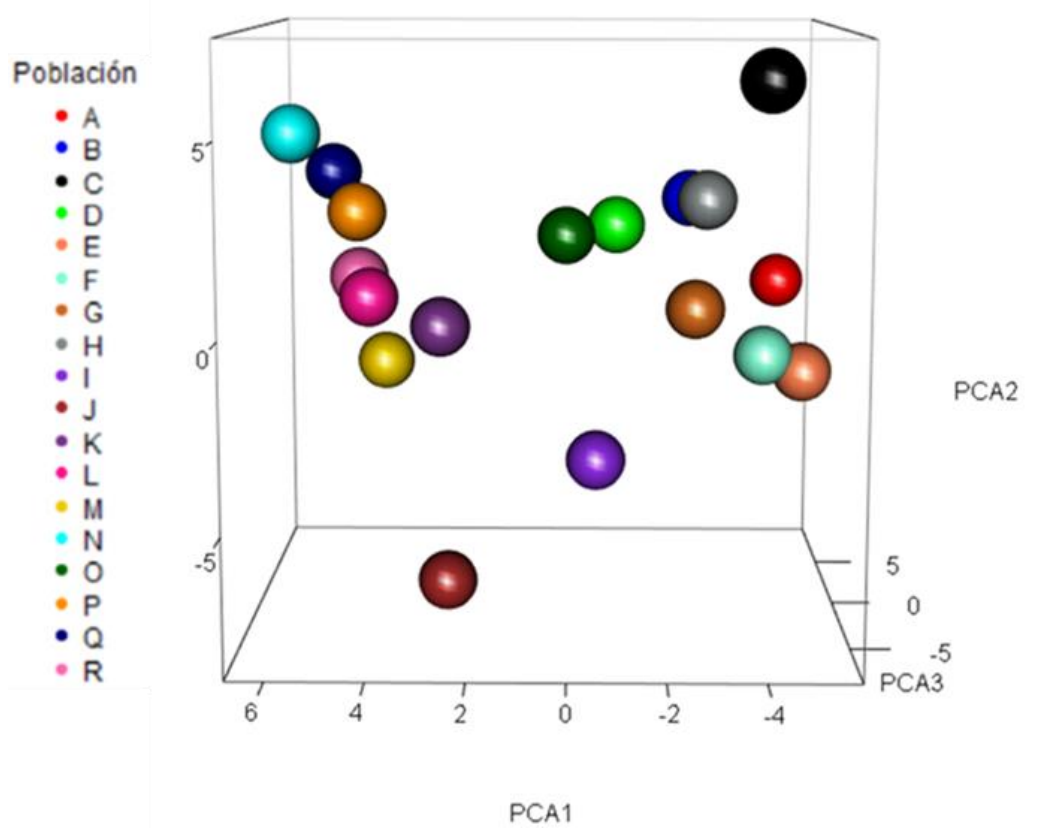

Cuadro 5: Valores propios y proporción de varianza explicada de los 7 componentes principales con la matriz de frecuencias de 109 alelos de poblaciones de gallinas

\begin{tabular}{|c|c|c|c|c|}
\hline $\mathbf{C P}$ & Valor propio & Proporción & Acumulada & Alelos \\
\hline & & & & $\begin{array}{l}\text { MCW32-A, MCW32-E, MCW94-B, } \\
\text { MCW94-D, MCW94-F, MCW134-A, } \\
\text { MCW135-C, MCW135-E, MCW135- }\end{array}$ \\
\hline & & & & MCW135-O, \\
\hline \multirow[t]{2}{*}{1} & 14.6493163 & 0.1344 & 0.1344 & MCW145-A, MCW95-A, MCW95-B \\
\hline & & & & $\begin{array}{l}\text { MCW158-B, MCW32-C, MCW32-D, } \\
\text { MCW32-G, MCW32-H, MCW94-B, } \\
\text { MCW114-K, MCW134-K, MCW135- }\end{array}$ \\
\hline \multirow[t]{3}{*}{2} & 10.7115308 & 0.0983 & 0.2327 & B, MCW135-J, MCW68-A \\
\hline & & & & $\begin{array}{l}\text { MCW131-C, MCW131-H, MCW158- } \\
\text { K, MCW94-A, MCW114-E, MCW134- }\end{array}$ \\
\hline & 9.7335336 & 0.0893 & 0.322 & $\begin{array}{l}\text { G, MCW134-J, MCW135-D, MCW95- } \\
\text { D, MCW95-H }\end{array}$ \\
\hline
\end{tabular}


Los agrupamientos obtenidos por el análisis de conglomerados (Figura 2) fueron similares a los observados en plano de dimensionalidad del análisis de componentes principales, se distinguen tres agrupaciones principales (distancia genética de 0.90). El primer grupo conformado por Nazareno, ITVO, San Lucas y San Antonio, que incluye poblaciones aisladas y diferentes genéticamente por características únicas de las gallinas criollas, producto de un reducido flujo genético en estas poblaciones criollas con poblaciones avícolas con un grado de recombinación con razas conocidas como Plymouth Rock. En el subgrupo 2 están las poblaciones que poseen un mayor grado de parentesco genético, evidenciado por la proporción de alelos con el grupo de referencia (Plymouth Rock), como causa del proceso de introducción de esta raza comercial en las comunidades mediante paquetes tecnológicos $\mathrm{y}$ en consecuencia, se origina un proceso de cruzamientos con gallinas adaptadas a las condiciones ambientales y de manejo de las diferentes zonas rurales, ocasionando una pérdida o desgaste de las características de adaptación y supervivencia de las gallinas criollas. El tercer subgrupo estuvo conformado por las poblaciones de Huixtepec 4, San Antonino 2, Teotitlán y Totolapam 1, comparten frecuencias alélicas similares, lo que sugiere que tienen una variabilidad en rasgos fenotípicos únicos, propios de las gallinas criollas.

Figura 2: Dendograma de las poblaciones de gallinas, construido con el método UPGMA con base en distancias euclidianas obtenidas a partir de frecuencias de 109 alelos de microsatélites

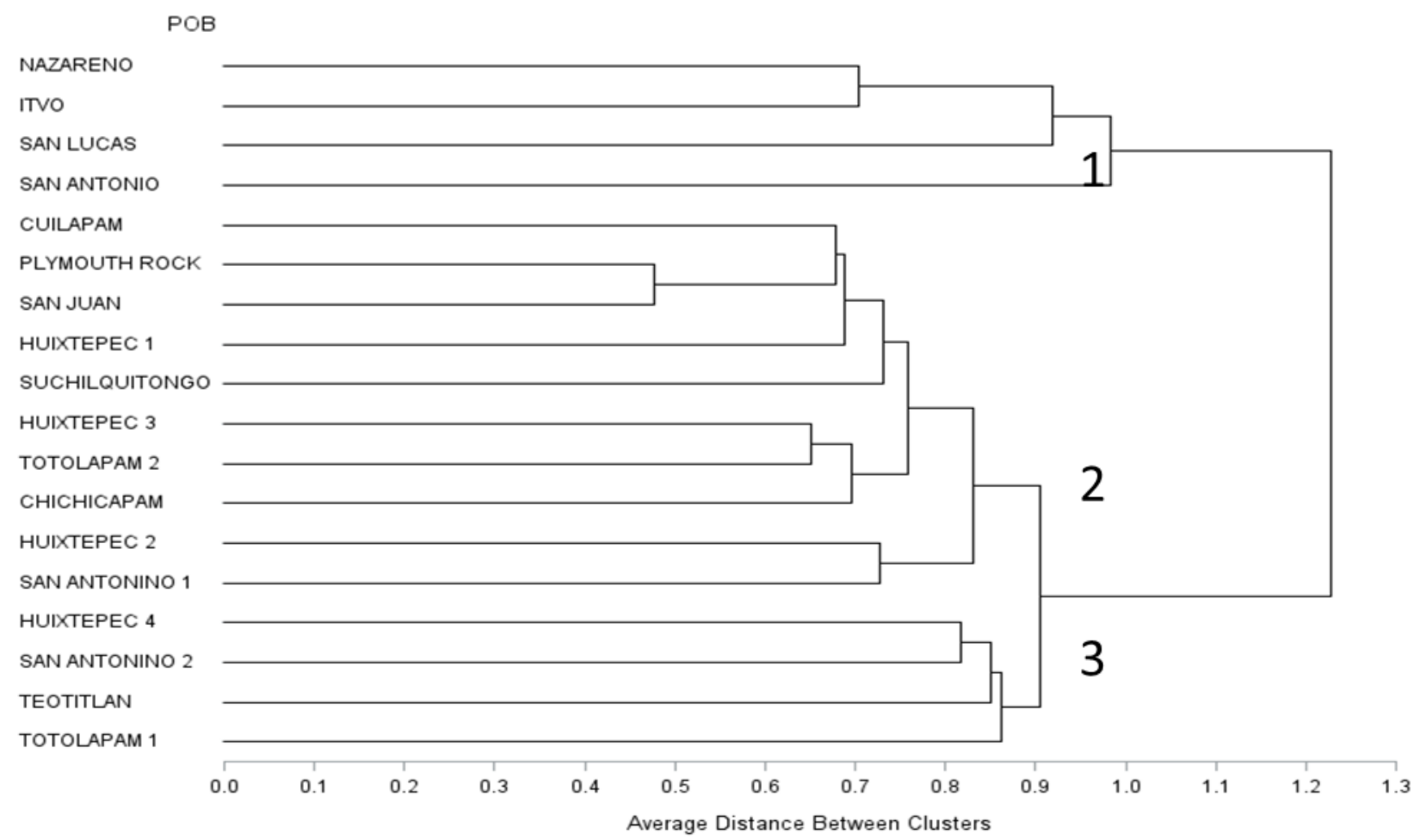




\section{Conclusiones e implicaciones}

Existe una amplia diversidad genética evidenciada por un total de 109 alelos detectados por los 10 microsatélites usados y un valor de Heterosigocidad esperada de 0.738 en las gallinas criollas de los Valles Centrales de Oaxaca. Los perfiles alélicos encontrados permitieron estimar un bajo grado de diferenciación entre individuos $\left(\mathrm{F}_{\mathrm{ST}}=0.059\right)$ entre las poblaciones localizadas en dichos s núcleos geográficos; en particular, la diversidad genética es más compleja en las poblaciones de Tlacolula (0.072) y Ocotlán (0.063). Una mínima proporción (5.9\%) de la diversidad genética total se localiza entre las poblaciones de gallinas criollas, mientras que el restante $94.1 \%$ reside dentro de las mismas.

\section{Literatura citada:}

1. Abebe AS, Mikko S, Johansson AM. Genetic diversity of five local Swedish chicken breeds detected by microsatellite markers. PLoS One. 2015;10(4):1-13.

2. FAO. La situación de los recursos zoogenéticos mundiales para la alimentación y la agricultura. Food Agric Organ United Nations. 2010.

3. FAO. Recursos genéticos animales 56. Food Agric Organ United Nations. 2015;171.

4. Kaya M, Yildiz MA. Genetic diversity among Turkish native chickens, Denizli and Gerze, estimated by microsatellite markers. Biochem Genet 2008;46(7-8):480-91.

5. Muir WM, Cheng HW. Genetic influences on the behavior of chickens associated with welfare and productivity. 2nd ed. Genetics and the behavior of domestic animals. Elsevier Inc. 2014. http://dx.doi.org/10.1016/B978-0-12-394586-0.00009-3.

6. Dorji N, Daungjinda M, Phasuk Y. Genetic characterization of Thai indigenous chickens compared with commercial lines. Trop Anim Health Prod 2011;43(4):779-85.

7. Qu L, Li X, Xu G, Chen K, Yang H, Zhang L, et al. Evaluation of genetic diversity in Chinese indigenous chicken breeds using microsatellite markers. Sci China, Ser C Life Sci 2006;49(4):332-341.

8. Sukhatme, PV. Sampling Theory of Surveys with Application. Iowa State College Press. Ames, Iowa. 1970.

9. Crooijmans RP, van Oers PA, Strijk JA, van der Poel JJ, Groenen MA. Preliminary linkage map of the chicken (Gallus domesticus) genome based on microsatellite markers: 77 new markers mapped. Poult Sci 1996;75(6):746-54. 
10. Ya-bo Y, Jin-yu W, Mekki DMM, Qing-Ping A, Hui-Fang L, Rong G, et al. Evaluation of genetic diversity and genetic distance between twelve Chinese indigenous chicken breeds based on microsatellite markers. Int J Poult Sci 2006;5(6):550-556.

11. Horbańczuk JO, Kawka M, Sacharczuk M, Cooper RG, Boruszewska K, Parada R, et al. A search for sequence similarity between chicken (Gallus domesticus) and ostrich (Struthio camelus) microsatellite markers. 2007;25(4):283-288.

12. SAS 9.4 Software. Institute Inc., Cary, North Carolina, USA. 2016.

13. Al-Qamashoui B, Simianer H, Kadim I, Weigend S. Assessment of genetic diversity and conservation priority of Omani local chickens using microsatellite markers. Trop Anim Heal Prod 2014;46:1-6.

14. Antos P, Andres K, Kapkowska E. Preliminary studies on genetic diversity of selected Polish local chicken varieties. J Cent Eur Agric 2013;14(1):11-22.

15. Bao WB, Shu JT, Wu XS, Musa HH, Ji CL, Chen GH. Genetic diversity and relationship between genetic distance and geographical distance in 14 Chinese indigenous chicken breeds and red jungle fowl. Czech J Anim Sci 2009;54(2):74-83.

16. Mukesh. Ruheena J, Uma G, Han J, Sathyakumar S. Cross-species applicability of chicken microsatellite markers for investigation of genetic diversity in Indian duck (Anas platyrhynchos) populations. African J Biotechnol. 2011;10(76):17623-17631.

17. Illel JH, Roenen MAMG, Reidlin PJF, Äki AM, Ortwijn MO. Biodiversity of 52 chicken populations assessed by microsatellite typing of DNA pools. Genet Sel Evol 2003;35:533-557.

18. Esfahani EN, Eskandarinasab MP, Khanian SE, Nikmard M, Molaee V. Genetic diversity of a native chicken breed in Iran. J Genet 2012;91:1-4.

19. Choi NR, Seo DW, Jemaa SB, Sultana H, Heo KN, Jo C, et al. Discrimination of the commercial Korean native chicken population using microsatellite markers. J Anim Sci Technol 2015;57(5):1-8.

20. Huo JL, Wu GS, Chen T, Huo HL, Yuan F, Liu LX, et al. Genetic diversity of local Yunnan chicken breeds and their relationships with red junglefowl. Genet Mol Res 2014;13(2):3371-3383.

21. Suh S, Sharma A, Lee S, Cho C, Kim J, Choi S. Genetic Diversity and Relationships of Korean Chicken Breeds Based on 30 Microsatellite Markers. Asian Australas J Anim Sci 2014;27(10):1399-1405. 
22. Yang K, Luo X, Wang Y, Yu Y, Chen Z. Eight trinucleotide microsatellite DNA markers from Tibetan chicken, Gallus gallus domesticus. Conserv Genet Resour 2009;1:225237.

23. Chatterjee RN, Bhattacharya TK, Dange M, Dushyanth K, Niranjan M, Reddy BLN, et al. Genetic heterogeneity among various Indigenous and other chicken populations with microsatellite markers. J Appl Anim Res 2015;43(3):266-271.

24. Yang K, Luo X, Wang Y, Yu Y, Chen Z. Ten polymorphic microsatellite loci in Tibetan chicken, Gallus gallus domesticus. Conserv Genet 2010;11(3):671-683.

25. Snyder L. Freifelder AD, Hartl DL. General genetics. UK: Jones and Bartlett publishers, Inc.;1985.

26. Ramadan S, Kayang BB, Inoue E, Nirasawa K, Hayakawa H. Evaluation of genetic diversity and conservation priorities for Egyptian chickens. Open J Anim Sci 2012;2(3):183-190.

27. Dorji N, Duangjinda M, Phasuk Y. Genetic characterization of Bhutanese native chickens based on an analysis of Red Jungle fowl (Gallus gallus gallus and Gallus gallus spadecieus), domestic Southeast Asian and commercial chicken lines (Gallus gallus domesticus). Genet Mol Biol 2012;35(3):603-609.

28. Ding FX, Zhang GX, Wang JY, Li Y, Zhang LJ, Wei Y, et al. Genetic diversity of a Chinese native chicken breed, Bian chicken, based on twenty-nine microsatellite markers. Asian-Australasian J Anim Sci 2010;23(2):154-161.

29. Zhao J, Li H, Kong X, Tang Z. Identification of single nucleotide polymorphisms in avian uncoupling protein gene and their association with growth and body composition traits in broilers. Can J Anim Sci 2006;345-350.

30. Noah O, Ngeranwa JJN, Binepal YS, Kahi AK, Bramwel WW, Ateya LO, et al. Genetic diversity of indigenous chickens from selected areas in Kenya using microsatellite markers. J Genet Eng Biotechnol 2017;15(2):489-495.

31. Kumar V, Mathew J, Sharma D, Kumar V, Shukla SK, Mathew J, et al. Genetic diversity and population structure analysis between Indian Red Jungle fowl and domestic chicken using microsatellite markers. Anim Biotechnol 2015;37-41. 